\title{
Baclofen therapy for chronic hiccup
}

\author{
C. Guelaud*, T. Similowski*, J-L. Bizec**, J. Cabane+, \\ W.A. Whitelaw ${ }^{++}$, J-P. Derenne*
}

Baclofen therapy for chronic hiccup. C. Guelaud, T. Similowski, J-L. Bizec, J. Cabane, W.A. Whitelaw, J-P. Derenne. (C)ERS Journals Ltd 1995.

ABSTRACT: Chronic hiccup is a rare but potentially severe condition, that can be symptomatic of a variety of diseases, or idiopathic. Many therapeutic interventions have been reported, most often as case reports. Among other drugs, baclofen has been suggested as a therapy for chronic hiccup. In a large series of patients, we have evaluated its therapeutic position.

In patients with chronic hiccup, defined as hiccup spell or recurring hiccup attacks lasting more than 7 days, investigation of the upper gastro-oesophageal tract (fibroscopy, manometry, and $\mathrm{pH}$ monitoring) was systematically performed. Most patients had tried numerous drugs in the past, without success. Baclofen was used as a first treatment in patients without evidence of any gastro-oesophageal disease $(n=17)$, and was undertaken only after full treatment of such disease $(n=55)$ had failed to solve the hiccup problem $(n=20)$. Baclofen has, therefore, been administered to 37 patients with chronic hiccup (average duration $4.6 \mathrm{yrs}$ ).

Baclofen produced a long-term complete resolution ( 18 cases) or a considerable decrease (10 cases) of hiccups in 28 of the 37 patients. There was no significant difference between patients with or without gastro-oesophageal disease.

We conclude that so-called idiopathic chronic hiccup often results from gastrooesophageal abnormalities. Also, if controlled studies confirm our encouraging results, baclofen can be a major element in the treatment of chronic hiccup that is idiopathic, or that cannot be helped by treatment of gastro-oesophageal diseases.

Eur Respir J., 1995, 8, 235-237.
*Service de Pneumologie, Groupe Hospitalier Pitié-Salpêtrière, Paris, France. **Service de Pneumologie, Hôpital Saint-Joseph, Paris, France. ${ }^{+}$Service de Médecine Interne, Hôpital Saint-Antoine, Paris, France. ++Dept of Medicine, University of Calgary, Alberta, Canada.

Correspondence: T. Similowski

Service de Pneumologie et de Réanimation Groupe Hospitalier Pitié-Salpêtrière

47-83 Bd de l'Hôpital

75761 Paris Cedex 13

France

Keywords: Baclofen

diaphragm

gastro-oesophageal reflux

hiccup

Received: May 311994

Accepted after revision November 61994
Hiccup is a complex neurorespiratory phenomenon, in which involuntary contraction of inspiratory muscles is associated with glottic closure and sound production. Trifling when transient, it can become a severe problem when chronic, resulting in insomnia, wasting, depression and even suicide (see [1] for review). Chronic hiccup $(\mathrm{CH})$ is a rare disorder, and the subject of sporadic case reports that give some idea of possible causes and anecdotal information about treatment. When no specific aetiology can be found, various nonspecific drug treatments are used. Among them, baclofen, a gammaaminobutyric acid (GABA) derivative [2], has occasionally been used with success [3]. In the present report, we provide data indicating that chronic hiccups are often associated with an oesophageal disorder, treatment of which can be efficacious. We also describe the results of systematic baclofen administration in patients for whom no cause had been found for the hiccup or who failed to respond to treatment of a documented oesophageal disease.

\section{Patients and Methods}

\section{Selection}

Patients were referred to the Groupe de Recherche et d'Investigation Cliniques du Hoquet Chronique de l'Adulte
(GRICHCA), a multidisciplinary group established in Paris in 1991 to study the pathophysiology and treatment of $\mathrm{CH}$.

$\mathrm{CH}$ has been defined by others as a hiccup spell lasting more than $48 \mathrm{~h}$, or recurring hiccup attacks $[4,5]$. In the present study, we arbitrarily chose 7 days instead of $48 \mathrm{~h}$.

\section{Investigations}

All patients were assessed by means of a thorough clinical examination aimed at detecting thoracic, abdominal, neurological or metabolic disorders known to cause hiccups. They routinely had a chest roentgenogram, serum electrolytes and creatinine.

The 37 patients reported in the present study belong to a series of 72 consecutive patients in whom no obvious explanation for the hiccup was found after the aforementioned investigations (in other words, there was no manifest evidence of significant chronic disease classically associated with hiccup - see table 3 in [1] - and in whom we systematically evaluated the upper gastrointestinal tract. This evaluation included endoscopy, 24 hour $\mathrm{pH}$ monitoring, and manometry. If a clear abnormality was present on one of these three tests, the remainder of the set was not always performed. 


\section{Treatment}

Baclofen was prescribed as the first step of hiccup therapy when gastro-oesophageal investigation did not show significant abnormalities (17 patients, Group I). If present, such abnormalities (frequently gastro-oesophageal reflux) were first vigorously treated with omeprazole and cisapride in full doses (55 patients); 35 patients responded completely to this treatment. When hiccup persisted after one week of this regimen (20 patients, Group II), the next step was to add baclofen, starting with $15 \mathrm{mg} \cdot \mathrm{day}^{-1}$ divided into three doses, and increasing by $15 \mathrm{mg} \cdot \mathrm{day}^{-1}$ every 3 days until the hiccups were controlled, or a maximum dose of $75 \mathrm{mg} \cdot \mathrm{day}^{-1}$ was reached, or side-effects became troublesome.

\section{Patients}

Age and sex. All patients but two (one in each group) were male. Mean age of all patients was $60 \pm 19$ (SD) yrs. Mean age for Group I was $61 \pm 20$ versus $58 \pm 19$ yrs for group II, not significantly different.

History of hiccup. Various histories of hiccup were described, from patients who came to the clinic because of a single bout lasting 7 days or more, to patients who had had recurrent long-lasting bouts for up to $30 \mathrm{yrs}$. Hiccup duration was at least one year in 26 out of 37 patients. Mean duration of hiccups before baclofen treatment was longer for Group II ( $6.5 \pm 8.0 \mathrm{yrs})$ than for group I (2.9 $\pm 3.1 \mathrm{yrs})$, but again not significantly different (Student's t-test).

Five patients, four of them in Group I, were treated because of a single episode of uninterrupted hiccups lasting 8-14 days, generally painful and incapacitating.

Maximal observed frequency was 35 hiccups. $\mathrm{min}^{-1}$, with a rough average value of 12 hiccups $\cdot \mathrm{min}^{-1}$. In no case did the description or observation of hiccups correspond to the diagnosis of diaphragmatic flutter.

History of hiccup treatment. Those patients who had had hiccups for months to years had usually tried to cure the problem with a variety of mechanical, psychological, and "alternative" medical treatments, as well as with standard pharmaceutical preparations, all of which had failed. Six patients had been prescribed seven or more drugs before being referred to the clinic, 11 had taken four or more drugs, and 21 had taken two or more. The most common of these drugs, in order of number of patients who had tried them, were metoclopramide (16 patients, daily dose 30-90 mg, duration 7-22 days), chlorpromazine (11 patients, daily dose $25-100 \mathrm{mg}$, duration 3-11 days), and amitriptyline (5 patients, daily dose 50-125 mg, duration 5-16 days). Various other substances, such as neuroleptics (e.g. haloperidol and sulpiride), benzodiazepines (e.g. diazepam, bromazepam or prazepam), calcium blockers (e.g. nifedipine) and antiarrhythmics (e.g. quinidine) could be found in the patients' treatment histories.

\section{Results}

Group I included 17 patients, of whom three were lost to follow-up before an assessment could be made of the success of treatment, leaving 14 cases for analysis. Group II included 20 patients, of which all were followed up.

In the following, "complete resolution" is defined as complete disappearance of hiccup, whereas "treatment failure" corresponds to a complete lack of change in hiccup frequency or intensity. "Partial resolution" denotes either hiccup transformation from continuous to intermittent, or a patient-reported decrease in frequency of about $50 \%$, or a patient-reported significant change in hiccup perception (e.g. disappearance of the painful nature of hiccups, or their markedly decreased intensity).

Of the 34 patients who returned to follow-up, 18 had complete resolution of their problem within a few days of beginning baclofen, and 10 more had a partial resolution. These patients themselves qualified the effect of treatment as considerable, if incomplete. Treatment failure was recorded in six patients. This gives a rate of complete resolution of $53 \%$ (49\% if the patients lost to follow-up are counted as failures), and a rate of partial response of $29 \%$ (27\% if the patients lost to follow-up are counted as failures). When separated into groups, the rate of complete resolution of hiccups was higher in Group I at $71 \%$ than in Group II at $40 \%$, but the difference did not reach statistical significance (Chi-squared $=1.79)$. There was also a higher rate of complete resolution $(83 \%)$ among those patients with hiccups lasting less than a year compared to those lasting a year or more $(46 \%)$, again not significant (Chi-squared $=1.09$ ).

In Group I, 7 out of 10 complete resolutions were obtained with $15 \mathrm{mg} \cdot \mathrm{day}^{-1}$ baclofen. Eight patients received higher doses, which were efficient in only three cases. In Group II, 4 out of 8 complete resolutions were obtained at $15 \mathrm{mg} \cdot \mathrm{day}^{-1}$, the other four being obtained by dose increase, suggesting the possibility of a dose-response phenomenon. However, among the 10 patients in this group where doses had to be raised above $15 \mathrm{mg} \cdot \mathrm{day}^{-1}$, only 4 complete resolutions were observed.

Baclofen was not associated with any adverse effects during the time of administration, except in the few patients needing more than $45 \mathrm{mg} \cdot$ day $^{-1}$. In these, nausea and somnolence were at times noted, but disappeared when doses were decreased.

\section{Discussion}

To the best of our knowledge, this study is the first to report the use of baclofen at a logical point in an algorithm for systematic diagnosis and treatment in a large series of patients with $\mathrm{CH}$. It illustrates the strong association between $\mathrm{CH}$ and gastro-oesophageal disease. Indeed, $76 \%$ of 72 patients investigated appeared to suffer such disease, which in most cases was clinically silent. It also shows that in patients who either have failed to respond to treatment of a gastro-oesophageal disease, or patients who do not have gastro-oesophageal disease and in whom hiccup is not symptomatic of a neural disease 
or a mediastinal process, baclofen is associated with either a substantial improvement or complete resolution of hiccups $82 \%$ of the time.

Because this was not a controlled study, it may be asked whether the disappearance of hiccups during administration of the drugs was only a coincidence or a placebo effect in some cases. We believe this to be very unlikely for the great majority of cases, who had had persistent hiccups for one to $30 \mathrm{yrs}$ and then saw them disappear or greatly improve during the few days when the baclofen dose was being increased. Also, most of the patients had had multiple treatments with other drugs, all of them unsuccessful (a situation which explains the frequent use of "intractable hiccup" to designate $\mathrm{CH}$ in the literature). Coincidental improvement is more possible for the five cases whose hiccups, whilst severe and persistent, had arisen de novo and lasted only a week or so before baclofen was given. It can be noted that one study attempted to compare baclofen and placebo and showed the superiority of the former [6]. In this very limited series (four cases) complete hiccup suppression was not obtained, probably because of the small doses used.

Various drugs, among which many have neural effects, are helpful in treatment of $\mathrm{CH}$. As comprehensively reviewed recently [1], success has been reported with chlorpromazine [7], metoclopramide [8], haloperidol, amitriptyline [9], carbamazepine, diphenylhydantoin, and valproic acid. Baclofen has also been used, and LAUNOIS et al. [1] reviewed about 15 case reports of baclofenrelated sustained suppression of $\mathrm{CH}[3,6,10-12]$. Baclofen is a GABA derivative, with presynaptic motor neurone inhibitory properties [2] at the spinal level, and postsynaptic central action. It has potent central antispastic properties, for which it is used in multiple sclerosis. Its mechanism of action in hiccup is unclear, but presumably central.

Although we do not have complete data on follow-up, the clinicians in charge of the patients' follow up have found that hiccup stops rapidly after the drug treatment has been initiated, but that a progressive decrease in hiccup frequency and amplitude over days is also possible. They agree that there is no need to prolong therapy for more than a few days if the treatment is ineffective, and that it is difficult to discern a dose-response pattern. In no case has hiccup relapse been observed with uninterrupted treatment. Drug withdrawal appears possible and should be attempted, because the hiccups may not relapse. If they do, reapplication of the drug has always been efficacious in our experience.

Although we cannot completely rule out contribution of a placebo effect, we do feel that these results strongly sug-

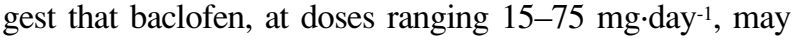
have potent action in chronic hiccup when no cause has been found, or when hiccups persist after treatment of a potentially causative oesophageal disease. Such disease should systematically be suspected in patients with $\mathrm{CH}$. Controlled studies remain necessary to fully describe baclofen efficacy.

\section{References}

1. Launois S, Bizec JL, Whitelaw WA, Cabane J, Derenne J-P. Hiccup in adults: an overview. Eur Respir J 1993; 6: $563-575$.

2. Davidoff RA. Antispasticity drugs: mechanisms of action. Ann Neurol 1985; 17: 107-116.

3. Burke AM, White AB, Brill N. Baclofen for intractable hiccup. N Engl J Med 1988; 319: 1354.

4. Souadjian JV, Cain JC. Intractable hiccup: etiological factors in 220 cases. Postgrad Med 1968; 43: 72-77.

5. Lewis JH. Hiccups: causes and cures. J Clin Gastroenterol 1985; 7: 539-552.

6. Ramirez FC, Graham DY. Treatment of intractable hiccup with baclofen: results of a double-blind randomized, controlled, cross-over study. Am J Gastroenterol 1992; 87: 1789-1791.

7. Friedgood CE, Ripstein CB. Chlorpromazine (thorazine) in the treatment of intractable hiccups. J Am Med Assoc 1955; 157: 309-310.

8. Madanagopolan N. Metoclopramide in hiccup. Curr Med Res Opin 1975; 3: 371-374.

9. Parvin R, Milo R, Klein C, Arlazoroff A. Amitryptiline for intractable hiccup. Am J Gastroenterol 1988; 63: $1007-1008$.

10. Lance JW, Bassil GT. Familial intractable hiccup relieved by baclofen. Lancet 1989; ii: 276-277.

11. Bhalotra R. Baclofen therapy for intractable hiccoughs (letter). J Clin Gastroenterol 1990; 12: 122.

12. Yaqoob M, Prabhu P, Ahmad R. Baclofen for intractable hiccups. Lancet 1989; ii: 562-563. 